\title{
Redheaded women are more sexually active than other women, but it is probably due to their suitors
}

Kateřina Sýkorováa a, , Vojtěch Fiala ${ }^{a}$, Jana Hlaváčováa ${ }^{a}$, Śárka Kaňková ${ }^{a}$, and Jaroslav Flegr ${ }^{a}$

${ }^{\text {a }}$ Department of Philosophy and History of Science, Faculty of Science, Charles University, Vinicna 7, 128 00, Prague, Czech Republic

"Corresponding author at: Department of Philosophy and History of Sciences, Faculty of Science, Charles University, Vinicna 7, 128 00, Prague, CZ

E-mail address: sykorok6@ natur.cuni.cz

\begin{abstract}
Women with red hair colour, i.e., 1-9\% of female Europeans, tend to be the subject of various stereotypes about their sexually liberated behaviour. The aim of the present casecontrol study was to explore whether a connection between red hair colour and sexual behaviour really exists using data from 110 women (34\% redheaded) and 93 men (22\% redheaded). Redheadedness in women, but not in men, correlated with various traits related to sexual life, namely with higher sexual desire as measured by Revised Sociosexual Orientation Inventory, with higher sexual activity and more sexual partners of the preferred gender over the past year, earlier initiation of sexual life, and higher sexual submissiveness. Structural equation modelling, however, showed that sexual desire of redheaded women meditated neither their higher sexual activity nor most of the variability of having more sexual partners. These results indicate that the apparently more liberated sexual behaviour in redheaded women could be the consequence of frequent attempts of potential mates to have sex with redheaded women. Other hypotheses, based on different physiology, faster life history strategy, or altered self-perception of red-haired women induced by stereotypes about them, were also tested and discussed.
\end{abstract}

Keywords: Redheadedness, Sexual desire, Sexual activity, Sexual submissiveness 


\section{Introduction}

Human redheadedness is determined by the quantity, ratio, and distribution of the two main types of the pigment melanin: eumelanin and pheomelanin. In European populations, its expression is controlled mainly by the $M C 1 R$ gene (Lin \& Fisher, 2007; Valverde et al., 1995). About 1-9\% of Europeans have red hair, with the highest prevalence in Great Britain (Katsara \& Nothnagel, 2019). Similar to other minorities, redheaded people are subjected to various stereotypes (Heckert \& Best, 1997). Redheaded women are thought of as being more temperamental than other women (Heckert \& Best, 1997; Swami \& Barrett, 2011; Weir \& Fine-Davis, 1989) and, unlike redheaded men, they are often stereotyped as sexy, passionate, sexually liberated, or promiscuous (Anderson, 2015; O'Regan, 2014). In scientific literature, however, data regarding any associations between traits related to sexual life and red hair are nearly absent. There are only studies showing higher self-reported lifelong number of sexual partners in redheaded women (Frost, Kleisner, \& Flegr, 2017), association between genetically predicted later age at first sexual intercourse and redheadedness in both sexes (Day et al., 2016), and no connection between self-reported sexual desire and redheadedness in either gender (Flegr \& Sýkorová, 2019). All these papers, however, primarily focused on different topics. The nature and cause of a possible relation between red hair and sexual behaviour in women therefore deserves a closer look.

Based on available scientific literature, we propose five principally distinct possible explanations of the potentially less restrained sexual behaviour in redheaded women. The hypothetical higher sexual activity of redheaded women could be either the result of their higher sexual desire or of a higher demand on the part of potential mates who prefer redheaded women or believe that redheaded women are more sexually permissive. If higher sexual desire of redheaded women were indeed responsible for their less restrained sexual behaviour, manifesting for instance in higher sexual activity or early initiation of sexual life, one would expect redheaded women to exhibit one of the following: (1) a difference in physiology, e.g. higher oestrogen concentrations; (2) a faster life history strategy, possibly induced by worse health that would shorten life expectancy and likely reproductive period; (3) their altered self-perception with internalised belief in stereotypes about redheads. If the higher sexual activity of redheaded women were the result of higher demand for women with red hair, it could be either (4) a direct passive response of redheaded women to current courtship of potential mates, or (5) indirect, partly or fully active, response of redheaded women to past courtship of potential mates. 
The first model supposes that redheaded women have on average higher sexual desire and sexual activity than non-redheaded women due to some molecular mechanism that affects both the expression of red hair in women and their sexual behaviour. The most probable candidate is prenatal oestrogen. Manning and Fink (2008) showed that women with a high 2D:4D digit ratio, which indicates exposure to a higher level of prenatal oestrogen (Manning et al., 1998), report higher sex drive and easier attainment of sexual excitement. Because red hair colour generally seems to occur more frequently in women than in men (Flegr \& Sýkorová, 2019; Frost et al., 2017; Shekar et al., 2008), Frost et al. (2017) suggested that prenatal oestrogen, which is specific to female development, contributes to the expression of red hair during prenatal development and might be responsible for the sex difference in the frequency of redheadedness. Conversely, a study by Voracek, Bagdonas, and Dressler (2007) found that prenatal oestrogen, as indirectly indicated by 2D:4D digit ratio, was not associated with lighter hair colours. Still, that study did not examine an association between estimated prenatal oestrogen and red hair colour specifically, because red hair colour was treated merely as one of the dark colours of hair. The sex difference in expression of red hair phenotype is, however, supported by a twin study which showed that in four of five twin pairs discordant in red hair colour, the females were redheaded and males non-redheaded (Box et al., 1997).

The second model suggests that higher sexual desire of redheaded women could be due to their switch from a slow to a faster life strategy, which could occur in response to poorer health. Redheaded people, especially women, tend to have worse health (Flegr \& Sýkorová, 2019; Frost et al., 2017) and are more likely to suffer from certain diseases including cancer, endometriosis, and Parkinson's disease (Bliss et al., 1995; Chen et al., 2017; Gao et al., 2009; Missmer et al., 2006; Scherer \& Kumar, 2010; Tell-Marti et al., 2015; Woodworth et al., 1995). It has been described how in reaction to impaired health, which reduces adult life expectancy and therefore also the length of the reproductive period, individuals tend to shift to a faster life history strategy, which manifests itself as earlier reproduction or having a higher number of children (Chua et al., 2017; Waynforth, 2012). A recent study has moreover shown that poor health is associated not only with an earlier start of reproduction but also with an earlier initiation of sexual life in women and higher sexual desire in both women and men (Sýkorová \& Flegr, 2021).

The third model proposes that redheaded women have higher sexual desire and are sexually more permissive because the stereotypes about their sexual behaviour have altered their self-perception. In this process, an initially incorrect attribution assigned by others can 
be internalised by the target person who then changes their self-perception to conform to the initially erroneous belief (Darley \& Fazio, 1980; McNulty \& Swann, 1994; Scherr et al., 2011; Snyder \& Swann, 1978). Based on such process of change in self-perception, redheaded women themselves could be internally convinced of the validity of the stereotypical social labelling, which would then lead to their actual higher sexual desire.

The fourth model puts potential mates, usually men, in the active role in explaining the association between women's redheadedness and their higher sexual activity. If men preferred redheaded women or, based on prevailing stereotypes, anticipated greater likelihood of success in their attempts to have sex with women with red hair, they would do so relatively more frequently. It would automatically lead to a higher sexual activity and a higher number of sexual partners of redheaded women even if their sexual desire was average or even lower than average.

The fifth model employs the principle of social feedback hypothesis (Kleisner et al., 2010) to explain the higher sexual activity in redheaded women. If potential mates tried to have sex with women with red hair often on the first date or early on in the courtship, redheaded women could start to consider it a social norm and begin to respond more positively to such behaviour or even initiate sexual activity themselves. Because redheaded women would perceive such behaviour as a social norm, they might be more sexually active even if they did not like it and were unfamiliar with stereotypes about more permissive redheaded women.

The main purpose of the present case-control study was to explore whether there is an association between red hair colour and traits related to sexual life. We collected data about hair colour, sexual behaviour, and sexual preferences from 110 women and 93 men with various intensity of redheadedness. We analysed the association of redheadedness with sexual desire, sexual activity, the number of sexual partners, age at first sexual intercourse, sexual orientation, BDSM preferences, and sexual dominance. In an attempt to test the several hypotheses explaining mechanism of the observed associations, we searched for a mediating role of sexual desire and physical disease.

\section{Material and methods}

The study consisted of a laboratory investigation which took place at the Faculty of Science of Charles University in Prague on 17 September - 3 October 2018 and subsequent online questionnaire survey performed with the same set of participants within the following few 
weeks. Information about the experimental design was already described in a study by Flegr et al. (2020). The project was approved by the institutional review board of the Faculty of Science, Charles University (No. 2018/30).

\section{Participants}

The recruitment of participants was conducted mostly via Facebook. The study was promoted as a 'study of health and personality of redheads' on the timeline of the Facebook page Labbunnies, an approximately 18,000-strong group of Czech and Slovak nationals willing to participate in evolutionary psychology experiments. Anyone could share the link to invitation to the study. Further recruitment of redheaded participants was carried out by invitations on other Facebook pages, selective invitation of registered members of Labbunnies who reported having red hair in our earlier questionnaires (scored 4-6 on a 6-point scale of redheadedness), and by handing out flyers in the streets of Prague to people who looked like natural redheads. We invited only people who did not have greying hair and had not dyed or bleached their hair for at least six months. The ratio of redheaded participants to controls was intended to be 1:2. In the end, we obtained data from 110 women and 93 men who participated in the study. All provided informed consent. Due to the sensitivity of questions posed to them, participants took part in the study under assigned anonymised codes and were informed about the complete anonymisation of the data. Participants received no remuneration, only a commemorative badge and a haircare gift set (costing 53 CZK, i.e., app. 2.3 USD). The data collection procedure and experimental design were preregistered prior to the beginning of the study on the website of the Open Science Framework (https://osf.io/p23dk).

\section{Variables}

In the project, redheadedness was determined using several methods: participants' self-report in the questionnaire, experimenters' observer report during the laboratory investigation, and two methods of measuring hair pigmentation with a spectrophotometer. A previously published study performed on the same data showed that all the above-mentioned indicators of redheadedness provide almost identical results (Flegr et al., 2020). In the present study, we employed only self-reported redheadedness because this way, the acquired results will be better comparable with past and future studies. Self-reported redheadedness was measured as a response to assessing current natural redheadedness on a six-point scale anchored with 'absolutely non-red' (code 1) and 'bright red' (code 6). Correlations between other indicators of redheadedness and sexual behaviours are presented in Supplementary Table S1, and again 
show that all methods of determining redheadedness provide highly similar results. It suggests that the simplest and cheapest method, namely participants' self-rating using a short ordinal scale, should be preferred in future studies because it facilitates acquisition of data from large population samples. Measurement scales and methods of calculating other indicators of redheadedness, which are presented in the Supplementary materials, are described in Flegr et al. (2020).

All output variables were obtained from the questionnaire data. Sexual desire was computed as the sum of Z-scores of three items forming the Desire facet in the Revised Sociosexual Orientation Inventory (Penke \& Asendorpf, 2008), namely the frequency of having fantasies about having sex with someone the respondent is not in a committed romantic relationship with, the frequency of experiencing sexual arousal when the respondent is in contact with someone who they are not in a committed romantic relationship with, and the frequency of having spontaneous fantasies about having sex with someone the respondent had just met (each item has been anchored with 'never' - code 1, and 'at least once a day' code 9). Sexual activity was estimated as the average number of sexual intercourse per month in the past year (eight categories: ' 0 ' - code 1, ' 1 ' - code 2, '2-3' - code 3, '4-6' - code 4, '7-10' - code 5, '11-20' - code 6, '21-30' - code 7, 'over 30' - code 8). Nonheterosexuality was calculated from two variables that inquired about the intensity of being attracted to people of the same and the opposite sex (anchored with 'absolutely not' - code 0 , and 'absolutely yes' - code 100; we intended to exclude any subjects who would respond with ' 0 ' on both scales but no such subject took part in the study). Non-heterosexuality was treated as a binary variable with 1 corresponding to being sexually attracted to people of the same sex with the same or higher intensity than to people of the opposite sex. In heterosexuals, we assessed the number of sexual partners of the preferred sex as the number of sexual partners of the opposite sex in the past year (nine categories: ' 0 ' - code 1, ' 1 ' code 2 , ' 2 ' - code 3 , ' 3 ' - code 4 , ' 4 ' - code 5, '5-6' - code 6, '7-9' - code 7, ' $10-19$ ' code 8, '20 or more' - code 9). In non-heterosexuals, the number of sexual partners of the preferred sex was expressed as the higher code of two variables assessing the number of female and male sexual partners in the past year (on the same scale as above). Age at first sexual intercourse was determined as age at such activity (restricted from below with ' 12 and less' - code 12, and restricted from above with '40 and more' - code 40; 'I haven't had sex yet.' corresponded to code 0). BDSM index was calculated as the arithmetical mean of Zscores of responses to questions concerning sexual preferences, namely the arousal by violence, own pain, placing oneself in danger, own powerlessness, own humiliation, other's 
pain, being in danger, powerlessness, and humiliation (each item was anchored with 'absolutely not' - code 0 , and 'absolutely yes' - code 100). Index of sexual dominance was computed as the arithmetical mean of Z-scores of 4 differences between arousal by other's and own pain, being in danger, powerlessness, and humiliation.

We also examined a number of potentially confounding variables including sex, age, size of place of residence (six categories: '<1,000 inhabitants', '1,000-5,000', '5,00050,000', ‘50,000-100,000', '100,000-500,000', 'Czech or Slovak capital') and current sexual partnership status (having a stable sexual partner, binary variable). Physical disease was calculated as the arithmetical mean of Z-scores of responses to the number of antibiotic treatments a respondent used in the past year, the number of different kinds of drugs prescribed by a medical doctor which a respondent uses daily, the number of different kinds of non-prescription drugs or food supplements a respondent uses on a daily basis, the number of visits to a general practitioner in the past year, the number of different medical specialists a respondent visited in the past year (each item had 1-9 scale), and the intensity of physical health problems (0-100 scale). Similarly, mental disease was computed as the arithmetical mean of Z-scores of responses to the intensity of suffering from anxieties, depressions, manias, obsessions, phobias, visual hallucinations, auditory hallucinations, burnout, and headaches, and the intensity of mental health problems (each item had 0-100 scale). Physical and mental disease were calculated from variables used for the computation of indices of physical and mental health problems in several previously published studies (Flegr \& Horáček, 2020; Flegr, Kuba, \& Kopecký, 2020). Variables pertaining to current health status were selected by a general practitioner (Flegr, Hoffmann, \& Dammann, 2015).

\section{Statistical analysis}

Before statistical analyses, we filtered out records of three subjects who had grey hair, their scalp was visible in the spot of measurement of hair colour, or who did not provide information about their age. When a respondent omitted over $20 \%$ of questions relevant for one index (i.e., sexual desire, BDSM index or index of sexual dominance), we did not compute the index for this subject and excluded their data from particular tests. In case missing data accounted for under $20 \%$ of variables relevant for a particular index, that index was calculated from the remaining variables.

The age of women and men was compared using the Wilcoxon test. Associations of all focal variables with sex and age were examined by the nonparametric Kendall corelation test. Associations of all focal variables with potentially confounding variables (i.e., size of 
place of residence, current sexual partnership status, physical disease, mental disease) were analysed by a partial Kendall correlation test with age as a covariate. We tested associations between redheadedness and traits related to sexual life using partial Kendall correlation test with age as a covariate. In the next step, we also used the same test with age and potentially confounding variables that had a significant effect on the output variables as covariates.

Partial Kendall correlation analysing the effect of redheadedness on the age at first sexual intercourse excluded 15 subjects who reported not having had their first sexual intercourse yet. To include the effect of those subjects, we conducted a Cox regression. Each univariate Cox regression model included the effect of redheadedness and age. Independent variables were standardised by computing Z-scores prior to the Cox regression. To investigate the role of potentially mediating variables in the association between redheadedness and sexual behaviour, we performed path analyses. To assess the strength of the observed effects, we used the widely accepted borders by Cohen (1977). After transformation between Tau and d, Taus $0.062,0.156$, and 0.241 correspond to d 0.20 (small effect), 0.50 (medium effect), and 0.80 (large effect), respectively (Walker, 2003). To address the issue of multiple testing, we applied the Benjamini-Hochberg procedure with false discovery rate set at 0.1 to the set of main tests. Statistical analysis was performed with $\mathrm{R} v$. 3.6.3 using the package 'explorer' 1.0 (Flegr \& Flegr, 2021) for analyses with partial Kendall correlation test, 'survival' 3.2.7 (Therneau, 2020) for computing Cox regression, and 'lavaan' 0.6-7 (Rosseel, 2012) and 'semPlot' 1.1.2 (Epskamp, 2015) for conducting path analysis. The dataset used in this article can be accessed on Figshare (https://doi.org/10.6084/m9.figshare.16790377.v1).

\section{Results}

The final set consisted of 109 women (mean age 27.3, SD 7.5) and 91 men (mean age 31.8, SD 8.8). The age of women and men differed significantly ( $\mathrm{W}=3,458, \mathrm{p}<0.001)$. On a sixpoint scale of self-reported redheadedness, 37 (33.9\%) women and 20 (22\%) men responded 4 and higher. Descriptive statistics for all variables used in this study are presented in Supplementary table S2.

We computed correlations of redheadedness and output variables with sex and potentially confounding variables (age, size of place of residence, current sexual partnership status, physical disease, and mental disease). Many focal variables significantly correlated with sex and age. For instance, women had more intensively red hair than men and 
redheadedness correlated negatively with age. Regarding other potential covariates, current sexual partnership status was significantly associated with four out of seven variables related to sexual behaviour. Size of place of residence, physical disease, and mental disease did not exhibit many significant associations with predictors and output variables (see Supplementary table S3).

Initially, we analysed the effect of redheadedness on variables related to sexual life with partial Kendall corelation controlled for age separately for women and men. In women, redheadedness positively corelated with sexual desire $(\tau=0.233, p<0.001, d=0.766)$, sexual activity $(\tau=0.306, \mathrm{p}<0.001, \mathrm{~d}=1.045)$, and the number of sexual partners of the preferred $\operatorname{sex}(\tau=0.286, \mathrm{p}<0.001, \mathrm{~d}=0.965)$, while corresponding negatively with age at first sexual intercourse $(\tau=-0.145, \mathrm{p}=0.039, \mathrm{~d}=0.465)$ and the index of sexual dominance $(\tau=-0.158, p=0.023, d=0.506)$. These results represent strong and medium effects (Cohen, 1977; Walker, 2003, also see Material and methods). Redheadedness in women did not show a significant correlation with non-heterosexuality $(\tau=-0.053, p=0.434, d=0.166)$ or with the BDSM index $(\tau=0.102, \mathrm{p}=0.141, \mathrm{~d}=0.323)$.

In men, redheadedness showed no significant correlation with any variable related to sexual life (sexual desire: $\tau=0.066, p=0.362, d=0.208$; sexual activity: $\tau=0.088, p=$ 0.226. $d=0.278$; the number of sexual partners of the preferred sex: $\tau=0.102, p=0.197, d=$ 0.324; age at first sexual intercourse: $\tau=0.082 ; \mathrm{p}=0.273, \mathrm{~d}=0.259$; non-heterosexuality: $\tau=$ 0.063, $\mathrm{p}=0.424$. $\mathrm{d}=0.199$; BDSM index: $\tau=0.110, \mathrm{p}=0.181, \mathrm{~d}=0.349$; index of sexual dominance: $\tau=0.035, p=0.676, d=0.111$ ). The results for both sexes remained qualitatively the same after correction by Benjamini-Hochberg procedure.

Current sexual partnership significantly corelated with sexual activity, the number of sexual partners of the preferred sex, non-heterosexuality, and BDSM index (Supplementary table S3). We have therefore repeated the analysis with partial Kendall correlation controlled for age and current sexual partnership. All results for women retained significance (sexual desire: $\tau=0.238, p<0.001$; sexual activity: $\tau=0.334, p<0.001$; the number of sexual partners of the preferred sex: $\tau=0.281, \mathrm{p}<0.001$; age at first sexual intercourse: $\tau=-0.146$, $p=0.039$; index of sexual dominance: $\tau=-0.157, p=0.025)$. For men, the results remained nonsignificant.

In our set, ten $(9.2 \%)$ women and five $(5.5 \%)$ men reported they had not yet had their first sexual intercourse. We excluded data from those subjects in the partial Kendall correlation testing the effect on age at first sexual intercourse, which, in theory, could lead to biased results. We have thus performed a Cox regression to include the effect of subjects who 
had not yet had their first sexual intercourse. In women, redheadedness showed a significant effect on the likelihood of having first sexual intercourse $(\mathrm{HR}=1.255, \mathrm{p}=0.033)$ suggesting this likelihood increases with women's redheadedness. In men, the likelihood of having first sexual intercourse was independent of redheadedness $(H R=1.146, p=0.232)$. The results of Cox regression therefore correspond to the results of partial Kendall correlation for both women and men.

The higher sexual activity of redheaded women could either result from their own higher sexual desire or be the consequence of a higher demand of potential mates for redheaded women (who are believed to be more sexually permissive). If the former is true, redheaded women's higher sexual activity should be mediated by their higher sexual desire, while if the latter is true, sexual desire will not play a mediating role and the intensity of sexual activity in redheaded women will not be associated with a higher intensity of sexual desire on their part. To discriminate between these two possibilities, we studied the effect of redheadedness on sexual activity and the number of sexual partners of the preferred sex using path analyses with sexual desire as a potentially mediating variable. In order to also test the hypothesis about higher sexual desire of redheaded women being a part of a faster life strategy (see Introduction), we included physical disease and age into the structured models. Redheadedness in women had direct positive effects on both sexual desire and sexual activity (Fig. 1, top left). The direct effect of redheadedness on sexual activity (standardised parameter estimate $=0.34$ ) was stronger than either the indirect (sexual desire-mediated) effect on sexual activity (standardised parameter estimate $=0.29 * 0.14=0.04$ ) or the direct effect on sexual desire (standardised parameter estimate $=0.29$ ). Similarly, women's redheadedness had direct positive effects on both sexual desire and the number of sexual partners of the preferred sex (Fig. 1, bottom left) and the direct effect of redheadedness on the number of sexual partners of the preferred sex (standardised parameter estimate $=0.33$ ) was likewise much stronger than the significant indirect effect of redheadedness (standardised parameter estimate $=0.31 * 0.24=0.07$ ).

These results indicate that the higher sexual activity and higher number of sexual partners of the preferred sex in redheaded women is not driven by their higher sexual desire. Path analysis also showed no effect of redheadedness on physical disease, nor any effect of worse health on sexual desire, sexual activity, or the number of sexual partners of the preferred sex. The explanation of higher sexual desire in redheaded women driven by adoption of a faster life strategy was not thus supported by the data. 
In men, redheadedness also tended to have a direct positive effect on sexual desire (Fig. 1, right), but it did not reach statistical significance (model for sexual activity: $p=0.26$; model for the number of sexual partners of the preferred sex: $p=0.052)$. Similarly, the results for men showed no effect of redheadedness on physical disease and the data thus provided no support for the faster life strategy hypothesis.

Women

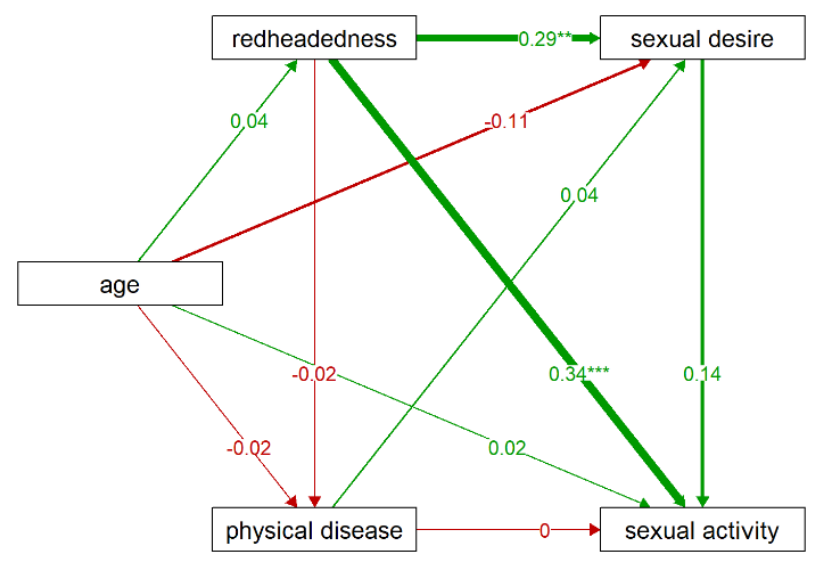

Women

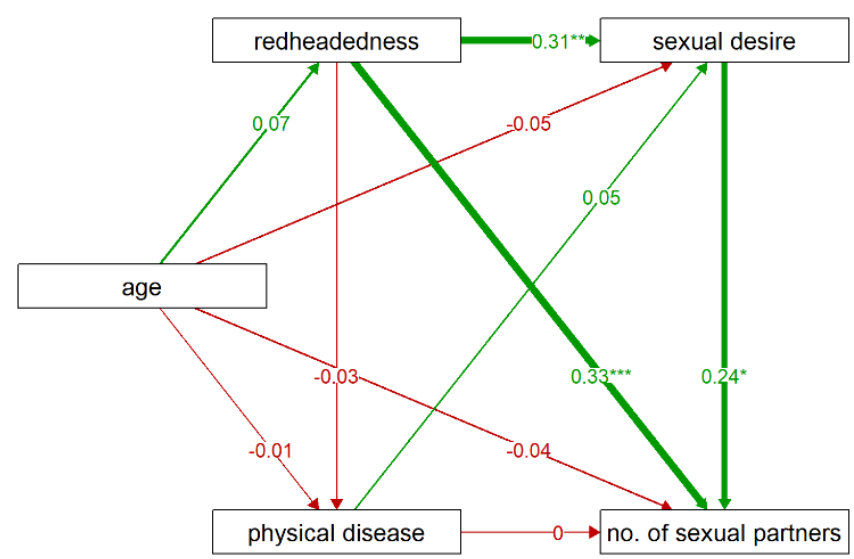

Men

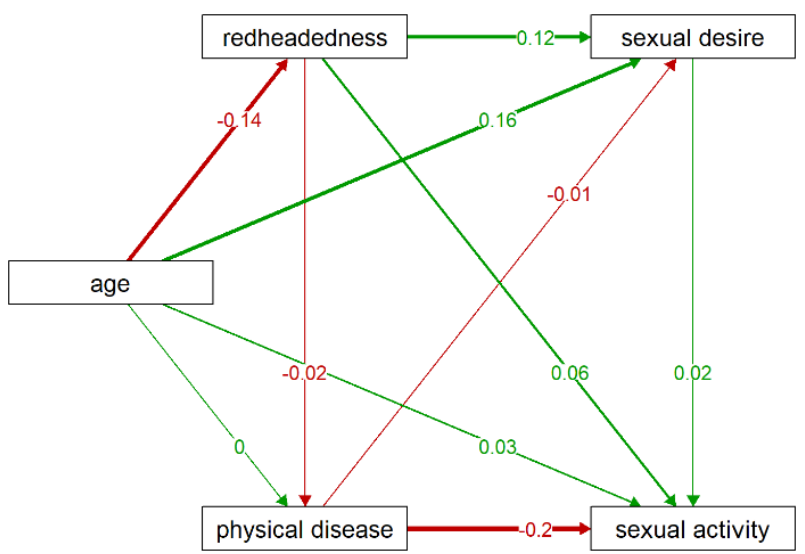

Men

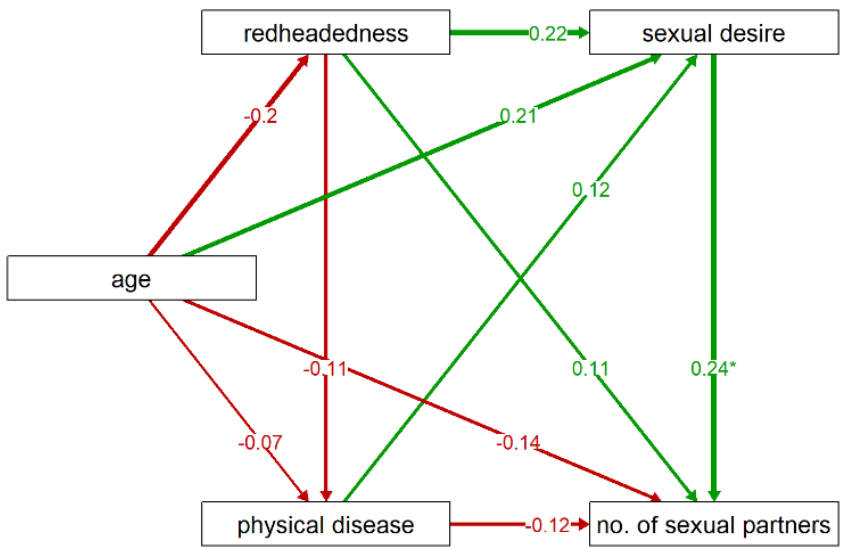

Figure 1. Direct and indirect effects of redheadedness on sexual activity and the number of sexual partners of the preferred sex in women and men. The figure visualises the results of path analyses of relations between redheadedness and sexual activity (top) and relations between redheadedness and the number of sexual partners of the preferred sex (bottom) separately for women (left) and men (right). Numbers at arrows show standardised parameter 
estimates. Associations with $p$ values under 0.05 are marked by ' $*$ ', those with $p$ values under 0.01 are marked by '**', and those with $\mathrm{p}$ values under 0.001 are marked by '***'.

\section{Discussion}

The results of this study showed that the intensity of redheadedness is associated with shifts in sexual behaviour in women but not in men. Women with red hair colour exhibited higher sexual desire and sexual activity, a higher number of sexual partners of the preferred sex, earlier initiation of sexual life, and lower sexual dominance (and therefore higher sexual submissiveness).

Our results are in accordance with recent findings of Frost et al. (2017), who reported a higher lifelong number of sexual partners in redheaded women. Similarly, our results correspond to the results of two studies that showed no relationship between hair colour (including red) and sexual orientation (Ellis et al., 2008; Loehlin \& McFadden, 2003). Contrary to our results, a large internet study by Flegr and Sýkorová (2019) found no significant correlation between self-reported red hair colour and sexual desire in either gender. In that study, however, sexual desire was measured as the intensity of being sexually attracted to people of the preferred sex (self-reported using a 1-100 scale), which might differ from sexual desire estimated more accurately by the SOI-R in the present study. Also inconsistent with our observations is a result by Day et al. (2016), who reported an association between genetically predicted red hair and later age at first sexual intercourse in both sexes. But that study involved subjects aged 40-69 years, while participants of the present study were at the peak of reproductive age, i.e., around 30. Therefore, this difference in results could be due to age cohort differences in initiation of sexual life and associated higher variability in the dataset of Day et al. (2016).

The higher sexual activity of redheaded women was not mediated by their higher sexual desire and the mediating effect of sexual desire in the association between redheadedness and number of sexual partners was weak. These observations thus do not support the first, second, and third model explaining sexual behaviour in redheaded women (see Introduction). It would seem that the fact that women have red hair more frequently than men do and, according to our data, exhibit more liberated sexual behaviour more often than men do might support the first model, which suggests the role of a molecule, e.g. prenatal oestrogen, that would affect both women's hair colour and sexual behaviour. This model also seems to find support in our results which show that redheaded women express higher sexual 
submissiveness, because the intensity of submissiveness is associated with a higher 2D:4D digit ratio that indicates exposure to prenatal oestrogen (Butovskaya et al., 2015; Manning \& Fink, 2008). However, if prenatal oestrogen were indeed responsible for higher sexual activity and higher number of sexual partners in women with red hair, it would affect their sexual desire, which would then mediate, to a large extent, their less restrained sexual behaviour. The existence of such a substantial mediating role of sexual desire was, however, excluded by the results of the path analysis.

Our results also do not support the second model, which proposes that sexual desire in redheaded women could be part of their faster life strategy induced by worse health. First of all, we found no correlation between redheadedness and physical disease in either sex and path analyses showed no mediation effect of worse health in associations between redheadedness and sexual desire. Secondly, redheaded women reported higher sexual activity and a higher number of sexual partners, while a previous study has shown that the faster life strategy induced by impaired health results in a higher sexual desire but lower sexual activity and having fewer sexual partners (Sýkorová \& Flegr, 2021). That study suggested that poor health adversely affects wellbeing and various physiological functions related to sexual life.

The third model, which aimed to explain higher sexual activity and sexual desire in redheaded women by their changed self-perception, is not supported by our analyses either. Because higher sexual desire did not mediate sexual activity in redheaded women, it is unlikely that their increased sexual activity is due to an altered self-concept that incorporates an internal conviction about the validity of stereotypes about permissive redheads.

The data of the present study do, however, seem to support the fourth model, which suggests that higher sexual activity and promiscuity of redheaded women result from their passive response to frequent mating efforts, usually made by men (who believe the stereotypes about permissive redheaded women). In redheaded women, a higher sexual activity and higher number of sexual partners were not primarily mediated by their higher sexual desire. In fact, the intensity of their sexual activity was relatively higher than the intensity of their sexual desire. This suggests that it is not the redheaded women's own initiative but higher demand for them which might be responsible for their higher sexual activity and higher number of sexual partners. It could be argued that red hair colour is often perceived as less attractive (Clayson \& Klassen, 1989; Clayson \& Maughan, 1976, 1986; Feinman \& Gill, 1978; Guéguen, 2012; Lawson, 1971; Swami \& Barrett, 2011). But Wortham, Miller, and Delvescovo (2018) showed that red hair was preferred over other hair colours more frequently than expected based on the prevalence of redheads in the studied 
population (both sexes preferred red hair $6 \%$ of the time, while only $3 \%$ of the female population were redheads). Given such imbalance between supply and demand, individual redheaded women are probably approached by potential mates more often than brunettes and blondes are.

To test this model in future, it would be useful to explore the sexual behaviour of women who have red body hair but are not redheaded. This would differentiate between active sexual behaviour of women with red hair and behaviour that is a passive consequence of mating efforts of potential suitors and prejudices about redheaded women. We could not conduct this investigation because our sample unfortunately contained only six nonredheaded women with red body hair.

We could neither prove nor disprove the fifth model, which suggests that the higher sexual activity of redheaded women is the result of a mechanism of social feedback. Based on our results, redheaded women might, in response to earlier experience with mating efforts, mostly made by males, consider higher sexual activity a social norm and react more positively in interaction with potential mates or even initiate sexual activity themselves. But to decide whether this mechanism applies, one would have to explore women's individual motivations and opinions related to sexual behaviour exhibited by women with and without red hair.

Although large cross-sectional internet studies showed worse health of redheaded individuals (Flegr \& Sýkorová, 2019; Frost et al., 2017), our data did not find this pattern. We have no explanation for the absence of this effect except that our laboratory experiments may have attracted a different subpopulation, namely people of lower age and in better physical and mental health (Flegr et al., 2020). A study made with the same sample of participants as the present study found that compared to non-redheaded individuals, redheaded subjects have higher concentrations of calcidiol, the precursor of vitamin $\mathrm{D}$, and their concentration of calcidiol seems independent of the intensity of sun exposure or protection from solar radiation (Flegr et al., 2020). That study suggested that people with red hair need less sun exposure to achieve satisfactory levels of vitamin D than non-redheaded people do. As a result, redheaded people might enjoy better health during some parts of the year, namely during autumn, winter, and spring, when the amount of UV-radiation in higher latitudes is low. At the same time, most health problems in redheaded individuals (oncological and gynaecological diseases, heart and vascular system problems, metabolic problems, fertility problems or Parkinson's disease, and the like) are more common in older, rather than young people. The mean age of participants of the present study (27.3 in women 
and 31.8 in men) was lower than that of respondents of previous studies exploring health in redheaded people (for instance, 32.9 and 34.6 in females and 35.1 and 36.8 in and Flegr and Sýkorová (2019) and Frost et al. (2017) respectively). The lower mean age of the present sample therefore might be why we did not find that redheaded subjects suffer from worse health. Moreover, the absence of any difference between the health status of redheaded and non-redheaded women in our study and the relatively young population sample could explain why we found in redheaded women higher sexual activity and a higher number of sexual partners of the preferred sex - which previous studies that were conducted on older samples did not report.

The main limitation of this study is that the participants did not form a random, representative sample of the Czech population. We suppose that subjects who took part in the laboratory investigation may have been exposed to a sieve effect, forming a group of rather altruistic, active people in good physical and mental condition. On the other hand, whenever people have the option of refusing to participate in a study, that is, in all studies performed in accordance with the widely accepted ethical standards, the issue of non-representativeness of a sample is always present. In any case, the observed results should be verified in future by using some more representative data, for instance collected by the internet questionnaire data, or by repeating the study on other samples that would not be self-selected for better health or altruism.

\section{Conclusion}

The results of the present study show that redheaded women exhibit higher sexual desire, higher sexual activity, a higher number of sexual partners, an earlier initiation of sexual life, and a higher level of sexual submissiveness. Because sexual desire does not seem to mediate the more liberated sexual behaviour in redheaded women in our data, stereotypical social labelling of redheaded women that might encourage potential mates to try and have sex with redheaded women more frequently is likely to account for the association between redheadedness and certain characteristics of sexual behaviour. In the process of forming this association, redheaded women can either affirmatively respond to current courtship of potential mates or consider frequent sexual activities a social norm and actively initiate sexual interactions themselves. Neither concentration of prenatal oestrogen, nor a faster life strategy induced by worse health or a strong internal belief of redheads in stereotypes about 
them is likely to be responsible for the association. Yet given the probably non-representative sample on which this study is based, our observations should be generalised with caution.

\section{Declarations}

Funding: The study was supported by the Grant Agency of Charles University (project no. 1494218).

Competing interests: The authors declare no competing interests.

Ethical approval: All procedures performed in studies involving human participants were in accordance with the ethical standards of the institutional research committee and with the 1964 Helsinki Declaration and its later amendments or comparable ethical standards. The study was approved by the institutional review board of the Faculty of Science, Charles University (No. 2018/30).

Consent to participate: Informed consent was obtained from all individual participants included in this study.

Availability of data: The dataset used in this article can be accessed on Figshare (https://doi.org/10.6084/m9.figshare.16790377.v1).

Authors' contributions: Kateřina Sýkorová designed research, collected and analysed the data, and wrote the manuscript. Vojtěch Fiala, Jana Hlaváčová, and Šárka Kaňková collected the data and revised the manuscript. Jaroslav Flegr designed research and participated in the interpretation of results and writing of the manuscript.

\section{Acknowledgement}

We wish to thank Anna Pilátová for final revisions of the manuscript.

\section{References}

Anderson, C. J. (2015). The importance of appearances in literature: What does it mean to be a redhead in literature? (bachelor's thesis). The University of Southern Mississippi.

Bliss, J. M., Ford, D., Swerdlow, A. J., Armstrong, B. K., Cristofolini, M., Elwood, J. M., . . . Easton, D. F. (1995). Risk of cutaneous melanoma-associated with pigmentation characteristics and freckling: Systematic overview of 10 case-control studies. International Journal of Cancer, 62(4), 367-376. doi:10.1002/ijc.2910620402 
Box, N. F., Wyeth, J. R., Ogorman, L. E., Martin, N. G., \& Sturm, R. A. (1997).

Characterization of melanocyte stimulating hormone receptor variant alleles in twins with red hair. Human Molecular Genetics, 6(11), 1891-1897. doi:10.1093/hmg/6.11.1891

Butovskaya, M., Burkova, V., Karelin, D., \& Fink, B. (2015). Digit ratio (2D:4D), aggression, and dominance in the Hadza and the Datoga of Tanzania. American Journal of Human Biology, 27(5), 620-627. doi:10.1002/ajhb.22718

Chen, X. Q., Chen, H. X., Cai, W. J., Maguire, M., Ya, B. L., Zuo, F. X., . . Schwarzschild, M. A. (2017). The melanoma-linked "redhead" MC1R influences dopaminergic neuron survival. Annals of Neurology, 81(3), 395-406. doi:10.1002/ana.24852

Chua, K. J., Lukaszewski, A. W., Grant, D. M., \& Sng, O. (2017). Human life history strategies: Calibrated to external or internal cues? Evolutionary Psychology, 15(1), 1474704916677342. doi:10.1177/1474704916677342

Clayson, D. E., \& Klassen, M. L. (1989). Perception of attractiveness by obesity and hair color. Perceptual and Motor Skills, 68(1), 199-202.

Clayson, D. E., \& Maughan, M. R. C. (1976). Blond is beautiful: Status and preference by hair color. Paper presented at the Annual meeting of the Rocky Mountain psychological association, Phoenix, Arizona.

Clayson, D. E., \& Maughan, M. R. C. (1986). Redheads and blonds: Stereotypic images. Psychological Reports, 59(2), 811-816.

Cohen, J. (1977). Statistical power analysis for the behavioral sciences (Rev. ed.). Academic Press.

Darley, J. M., \& Fazio, R. H. (1980). Expectancy confirmation processes arising in the social interaction sequence. American Psychologist, 35(10), 867-881. doi:10.1037/0003066x.35.10.867

Day, F. R., Helgason, H., Chasman, D. I., Rose, L. M., Loh, P. R., Scott, R. A., . . Perry, J. R. B. (2016). Physical and neurobehavioral determinants of reproductive onset and success. Nature Genetics, 48(6), 617-623. doi:10.1038/ng.3551

Ellis, L., Ficek, C., Burke, D., \& Das, S. (2008). Eye color, hair color, blood type, and the rhesus factor: Exploring possible genetic links to sexual orientation. Archives of Sexual Behavior, 37(1), 145-149. doi:10.1007/s10508-007-9274-0

Epskamp, S. (2015). semPlot: Unified visualizations of structural equation models. Structural Equation Modeling: A Multidisciplinary Journal, 22(3), 474-483.

doi:10.1080/10705511.2014.937847 
Feinman, S., \& Gill, G. W. (1978). Sex differences in physical attractiveness preferences. The Journal of Social Psychology, 105(1), 43-52. doi:The Journal of Social Psychology, Vol. 105, No. 1, June 1978: pp. 43-52

Flegr, J., \& Flegr, P. (2021). Doing exploratory analysis in R with a package Explorer v. 1.0. figshare. doi:10.6084/m9.figshare.14685825.v1

Flegr, J., Hoffmann, R., \& Dammann, M. (2015). Worse health status and higher incidence of health disorders in Rhesus negative subjects. Plos One, 10(10), e0141362. doi:10.1371/journal.pone.0141362

Flegr, J., \& Horáček, J. (2020). Negative effects of latent toxoplasmosis on mental health. Frontiers in Psychiatry, 10(1012). doi:10.3389/fpsyt.2019.01012

Flegr, J., Kuba, R., \& Kopecký, R. (2020). Rhesus-minus phenotype as a predictor of sexual desire and behavior, wellbeing, mental health, and fecundity. Plos One, 15(7), e0236134. doi:10.1371/journal.pone.0236134

Flegr, J., \& Sýkorová, K. (2019). Skin fairness is a better predictor for impaired physical and mental health than hair redness. Scientific Reports, 9, 13. doi:10.1038/s41598-019$54662-5$

Flegr, J., Sýkorová, K., Fiala, V., Hlaváčová, J., Bičíková, M., Máčová, L., \& Kaňková, Š. (2020). Increased 25(OH)D3 level in redheaded people: Could redheadedness be an adaptation to temperate climate? Experimental Dermatology, 29(7), 598-609. doi:10.1111/exd.14119

Frost, P., Kleisner, K., \& Flegr, J. (2017). Health status by gender, hair color, and eye color: Red-haired women are the most divergent. Plos One, 12(12), 16. doi:10.1371/journal.pone.0190238

Gao, X., Simon, K. C., Han, J., Schwarzschild, M. A., \& Ascherio, A. (2009). Genetic determinants of hair color and Parkinson's disease risk. Annals of Neurology, 65(1), 76-82. doi:10.1002/ana.21535

Guéguen, N. (2012). Hair color and courtship: Blond women received more courtship solicitations and redhead men received more refusals. Psychological Studies, 57(4), 369-375. doi:10.1007/s12646-012-0158-6

Heckert, D. M., \& Best, A. (1997). Ugly duckling to swan: Labeling theory and the stigmatization of red hair. Symbolic Interaction, 20(4), 365-384. doi:10.1525/si.1997.20.4.365 
Katsara, M. A., \& Nothnagel, M. (2019). True colors: A literature review on the spatial distribution of eye and hair pigmentation. Forensic Science International-Genetics, 39, 109-118. doi:10.1016/j.fsigen.2019.01.001

Kleisner, K., Kočnar, T., Rubešová, A., \& Flegr, J. (2010). Eye color predicts but does not directly influence perceived dominance in men. Personality and Individual Differences, 49(1), 59-64. doi:10.1016/j.paid.2010.03.011

Lawson, E. D. (1971). Hair color, personality and the observer. Psychological Reports, 28(1), 311-322. doi:10.2466_pr0.1971.28.1.311

Lin, J. Y., \& Fisher, D. E. (2007). Melanocyte biology and skin pigmentation. Nature, 445(7130), 843-850. doi:10.1038/nature05660

Loehlin, J. C., \& McFadden, D. (2003). Otoacoustic emissions, auditory evoked potentials, and traits related to sex and sexual orientation. Archives of Sexual Behavior, 32(2), 115-127. doi:10.1023/a:1022496207882

Manning, J. T., \& Fink, B. (2008). Digit ratio (2D:4D), dominance, reproductive success, asymmetry, and sociosexuality in the BBC Internet Study. American Journal of Human Biology, 20(4), 451-461. doi:10.1002/ajhb.20767

Manning, J. T., Scutt, D., Wilson, J., \& Lewis-Jones, D. I. (1998). The ratio of 2nd to 4th digit length: a predictor of sperm numbers and concentrations of testosterone, luteinizing hormone and oestrogen. Human Reproduction, 13(11), 3000-3004. doi:10.1093/humrep/13.11.3000

McNulty, S. E., \& Swann, W. B. (1994). Identity negotiation in roommate relationships: The self as architect and consequence of social reality. Journal of Personality and Social Psychology, 67(6), 1012-1023. doi:10.1037/0022-3514.67.6.1012

Missmer, S. A., Spiegelman, D., Hankinson, S. E., Malspeis, S., Barbieri, R. L., \& Hunter, D. J. (2006). Natural hair color and the incidence of endometriosis. Fertility and Sterility, 85(4), 866-870. doi:10.1016/j.fertnstert.2005.12.008

O'Regan, K. (2014). Red hair in popular culture and the relationship with anxiety and depression. (bachelor's thesis). University College Cork, Ireland.

Penke, L., \& Asendorpf, J. B. (2008). Beyond global sociosexual orientations: A more differentiated look at sociosexuality and its effects on courtship and romantic relationships. Journal of Personality and Social Psychology, 95(5), 1113-1135. doi:10.1037/0022-3514.95.5.1113

Rosseel, Y. (2012). lavaan: An R package for structural equation modeling. Journal of Statistical Software, 48(2), 1-36. 
Scherer, D., \& Kumar, R. (2010). Genetics of pigmentation in skin cancer - A review. Mutation Research - Reviews in Mutation Research, 705(2), 141-153. doi:10.1016/j.mrrev.2010.06.002

Scherr, K. C., Madon, S., Guyll, M., Willard, J., \& Spoth, R. (2011). Self-verification as a mediator of mothers' self-fulfilling effects on adolescents' educational attainment. Personality and Social Psychology Bulletin, 37(5), 587-600. doi:10.1177/0146167211399777

Shekar, S. N., Duffy, D. L., Frudakis, T., Montgomery, G. W., James, M. R., Sturm, R. A., \& Martin, N. G. (2008). Spectrophotometric methods for quantifying pigmentation in human hair - influence of MC1R genotype and environment. Photochemistry and Photobiology, 84(3), 719-726. doi:10.1111/j.1751-1097.2007.00237.x

Snyder, M., \& Swann, W. B. (1978). Behavioral confirmation in social interaction: From social perception to social reality. Journal of Experimental Social Psychology, 14(2), 148-162. doi:10.1016/0022-1031(78)90021-5

Swami, V., \& Barrett, S. (2011). British men's hair color preferences: An assessment of courtship solicitation and stimulus ratings. Scandinavian Journal of Psychology, 52(6), 595-600. doi:10.1111/j.1467-9450.2011.00911.x

Sýkorová, K., \& Flegr, J. (2021). Faster life history strategy manifests itself by lower age at menarche, higher sexual desire, and earlier reproduction in people with worse health. Scientific Reports, 11(1), 18. doi:10.1038/s41598-021-90579-8

Tell-Marti, G., Puig-Butille, J. A., Potrony, M., Badenas, C., Milà, M., Malvehy, J., . . Puig, S. (2015). The MC1R melanoma risk variant p.R160W is associated with Parkinson disease. Annals of Neurology, 77(5), 889-894. doi:10.1002/ana.24373

Therneau, T. (2020). A package for survival analysis in R. R package version 3.2-7. Retrieved from https://CRAN.R-project.org/package=survival

Valverde, P., Healy, E., Jackson, I., Rees, J. L., \& Thody, A. J. (1995). Variants of the melanocyte-stimulating hormone receptor gene are associated with red hair and fair skin in humans. Nature Genetics, 11(3), 328-330. doi:10.1038/ng1195-328

Voracek, M., Bagdonas, A., \& Dressler, S. G. (2007). Digit ratio (2D:4D) in Lithuania once and now: Testing for sex differences, relations with eye and hair color, and a possible secular change. Collegium Antropologicum, 31(3), 863-868.

Walker, D. A. (2003). JMASM9: Converting Kendall's Tau for correlational or meta-analytic analyses. Journal of Modern Applied Statistical Methods, 2(2), 525-530. doi:10.22237/jmasm/1067646360 
Waynforth, D. (2012). Life-history theory, chronic childhood illness and the timing of first reproduction in a British birth cohort. Proceedings of the Royal Society B-Biological Sciences, 279(1740), 2998-3002. doi:10.1098/rspb.2012.0220

Weir, S., \& Fine-Davis, M. (1989). 'Dumb blonde' and 'temperamental redhead': The effect of hair colour on some attributed personality characteristics of women. The Irish Journal of Psychology, 10(1), 11-19.

Woodworth, S. H., Singh, M., Yussman, M. A., Sanfilippo, J. S., Cook, C. L., \& Lincoln, S. R. (1995). A prospective study on the association between red hair color and endometriosis in infertile patients. Fertility and Sterility, 64(3), 651-652. doi:10.1016/S0015-0282(16)57809-1

Wortham, J., Miller, A., \& Delvescovo, D. (2018). Male and female hair color preferences: influences of familiarity, geographic region of origin, and environment on mate attraction in University of Tampa students. Florida Scientist, 81(1), 33-54. 